\title{
植物有性生殖对大气 $\mathrm{CO}_{2}$ 浓度变化响应的研究进展
}

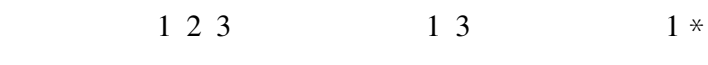 \\ (1 中国科学院植物研究所光合作用与环境分子生理学重点实验室, 北京 100093) \\ （2 南京农业大学园艺学院, 南京 210095) (3 中国科学院研究生院, 北京 100049)
}

\begin{abstract}
摘 要 比较详细地概述了过去数十年关于在大气 $\mathrm{CO}_{2}$ 浓度升高条件下 植物有性生殖特性发生变化的主要研究 成果。随着植物相对生长速率加快, 植株达到有性生殖所需形体大小的时间变短, 开花期提前, 生殖器官的生物量 也相应提高 其主要表现为开花数量、花粉和花蜜产量、果实数量与大小、种子大小与产量等均有不同程度的增加。 对大多数农作物而言, 种子产量的增加主要通过种子数量的增加, 而与种子大小变化关系不大。通常, 高浓度 $\mathrm{CO}_{2}$ 对豆科植物种子含氮量影响比较小, 却能显著地降低非豆科植物种子含氮量。不同类型植物的生殖生物量增加趋 势存在一定的规律性, 如不定型植物 > 定型植物, 豆科植物 > $\mathrm{C}_{3}$ 非豆科植物 > $\mathrm{C}_{4}$ 植物, 栽培植物 > 野生植物。针 对国内外对 $\mathrm{CO}_{2}$ 浓度升高影响植物有性生殖特性的研究中存在的不足, 该文提出了今后研究应该注意的问题。

关键词 $\mathrm{CO}_{2}$ 浓度升高 有性生殖 植物响应 种子质量 生殖特性
\end{abstract}

\section{A REVIEW ON RESPONSES OF PLANT SEXUAL REPRODUCTION TO ELEVATED $\mathrm{CO}_{2}$}

\author{
TENG Nian-Jun ${ }^{1,2,3}$ CHEN Tong ${ }^{1,3}$ and LIN Jin-Xing ${ }^{1 *}$
}

(1 Key Laboratory of Photosynthesis and Environmental Molecular Physiology , Institute of Botany , Chinese Academy of Sciences , Beijing 100093 , China)

(2 College of Horticulture, Nanjing Agricultural University, Nanjing 210095, China)

(3 Graduate University of Chinese Academy of Sciences , Beijing 100049 , China)

\begin{abstract}
Plant reproductive traits are key characteristics for predicting the impacts of global changes on plant community, agro-ecosystem and plant ecological fitness. This review seeks to integrate current results about the effects of elevated $\mathrm{CO}_{2}$ on plant reproductive traits in detail based on the existing experimental data in the past few decades. Earlier investigations demonstrate that elevated $\mathrm{CO}_{2}$ advances the flowering time through increasing relative growth rate and accelerating developmental process. All the numbers of flowers, fruits, and seeds , the mass of seed as well as the production of pollen and nectar of plants have been found to be stimulated by $\mathrm{CO}_{2}$ enrichment. It is further revealed that the increase in plant yields results largely from an increase in seed number rather than from individual seed mass. Elevated $\mathrm{CO}_{2}$ concentrations have little effect on seed [ $\mathrm{N}$ ] of legumes, but significantly reduce seed $[\mathrm{N}]$ of most nonlegumes. Contents of proteins , amino acids , and some mineral ions usually decrease in seeds of most nonlegumes. Different functional groups of plants are often found to differ markedly in their reproductive responses to elevated $\left.\mathrm{CO}_{2}: 1\right)$ crops allocate more mass to reproduction and produce more fruits and seeds than do undomesticated species ;2) indeterminate plants have stronger responses to elevated $\mathrm{CO}_{2}$ in comparison with determinate species ; 3 ) legumes are most responsive to elevated $\mathrm{CO}_{2}$, followed by nonlegume $\mathrm{C}_{3}$ species , then $\mathrm{C}_{4}$ species. Based on the data available , it seems rational to believe that changes in plant reproductive traits resulting from elevated $\mathrm{CO}_{2}$ may alter the competitive hierarchy , the species composition, and hence the functioning of plant community. Finally, some issues noteworthy for future researches in this field are also put forward with reference to the existing unsolved questions.
\end{abstract}

Key words Elevated $\mathrm{CO}_{2}$, Sexual reproduction, Plant responses, Seed quality, Reproductive traits

工业革命以来, 随着化石燃料消耗的不断增加 以及伐木毁林的加剧，全球大气 $\mathrm{CO}_{2}$ 浓度从当时的 $280 \mu \mathrm{mol} \mathrm{mol}^{-1}$ 增加到目前的 $370 \mu \mathrm{mol} \mathrm{mol}^{-1}$ 左右, 并且还在以每年 $1.5 \mu \mathrm{mol} \mathrm{mol}^{-1}$ 的速率递增, 预计
到本世纪末,大气 $\mathrm{CO}_{2}$ 浓度将会增加到 $540 \sim 970$ $\mu \mathrm{mol} \mathrm{mol}^{-1}($ IPCC, 2001$)$ 。自 20 世纪 70 年代以来， 有关大气 $\mathrm{CO}_{2}$ 浓度增高及其全球变化, 一直为全世 界关注的研究热点之一(张新时等, 1997; 方精云, 
2000)。近十多年来, 关于大气 $\mathrm{CO}_{2}$ 浓度升高对植物 的影响，国内外学者曾从分子、细胞、器官、物种、种 群直至生物圈等不同层次分别做了大量的研究工 作 发表的学术论文已达数千篇之多, 其中还有不少 评述性的文章 (Mortensen , 1987 ; Lawlor \& Mitchell， 1991 ;Idso \& Idso,1994;林金星等,1993; 蒋高明， 1995 蒋高明等,1997;汪杏梅等,1997;杨金艳等， 2002)。在这些评述性文章中, 主要涉及 $\mathrm{CO}_{2}$ 浓度升 高对植物光合与呼吸强度、叶绿素含量、营养器官各 种生长参数和结构、气孔导度与密度、水分利用效 率、生物量与产量、植物化学成分以及主要酶系统含 量等方面的影响，但却很少涉及专门评述植物有性 生殖对 $\mathrm{CO}_{2}$ 浓度变化的响应。然而值得注意的是， 植物有性生殖与其生态适应性, 以及与农作物籽粒 产量的关系极为密切。此外, 植物有性生殖特性的 变化,也可作为预测植物对全球气候变化响应的重 要指标之一 (Ziska \& Caulfield ,2000 ;Jablonski et al. , 2002)。为此, 本文将全面综述近十多年来, 有关大 气 $\mathrm{CO}_{2}$ 浓度升高对植物有性生殖变化特点的国内外 报道，以期为国内正在或即将开展这方面的研究工 作提供参考。

\section{1 对植物开花时间的影响}

通常在高浓度 $\mathrm{CO}_{2}$ 条件下, 大多数植物开花会 提前数天到两周不等 (Garbutt \& Bazzaz ,1984; Murray，1997)。一些重要农作物如小麦( Triticum aestivum)、水稻( Oryza sativa)、大豆 (Glycine max) 等在高 浓度 $\mathrm{CO}_{2}$ 条件下，均比对照提前数天开花 (Miglietta et al .1998; Uprety et al .,2003 ;Johannessen et al. , $2005)$ 。Wagner 等(2001)研究发现, 优质牧草白三叶 (Trifolium repens) 在 $600 \mu \mathrm{mol} \mathrm{mol}^{-1} \mathrm{CO}_{2}$ 浓度条件 下, 开花时间比正常的提前 $10 \mathrm{~d}$, 作者认为可能由于 其花芽分生组织在发育过程中获得了更多的营养物 质, 使发育速度加快, 从而开花时间提前。拟南芥 (Arabidopsis thaliana) 在 $1000 \mu \mathrm{mol} \mathrm{mol}{ }^{-1} \mathrm{CO}_{2}$ 浓度处 理下 $32 \mathrm{~d}$ 时抽苔, 而在 $360 \mu \mathrm{mol} \mathrm{mol}^{-1} \mathrm{CO}_{2}$ 浓度下 $37 \mathrm{~d}$ 时才抽苔, 通常在播种后的 3 周内, 正是拟南芥 幼苗生长发育的关键阶段, 它对 $\mathrm{CO}_{2}$ 浓度升高的反 应最为敏感, 生长明显加快, 而 3 周以后其相对生长 速率则无明显变化 (Gibeaut et al .,2001)。相反地, 有些植物开花时间对高浓度 $\mathrm{CO}_{2}$ 处理反应不明显, 甚至会推迟。如黑麦草 (Lolium perenne) 开花时间不 受高浓度 $\mathrm{CO}_{2}$ 处理的影响 (Wagner et al.,2001) ;而 菊花 (Dendranthema grandiflora) 随着 $\mathrm{CO}_{2}$ 浓度的增加
则延长了开花时间 (Reekie et al，1994)。由上可 见，在大气 $\mathrm{CO}_{2}$ 浓度升高条件下，各种植物的开花时 间均存在明显差异, 这可能与物种的光周期特性密 切相关。如长日照植物个体发育、花芽的诱导和发 育均受到高浓度 $\mathrm{CO}_{2}$ 的影响相应提前开花; 而短日 植物的花芽诱导则被推迟，或延缓花芽发育的时间 (Reekie et al.,1994 ; Ellis et al. ,1995 ; Rusterholz \& Erhardt , 1998 ; Ladeau \& Clark , 2001 ; Wagner et al . , 2001 ;Lewis et al .,2003)。Reekie 等(1994)曾报道了 $\mathrm{CO}_{2}$ 浓度加倍分别对菊花、长寿花 (Kalanchoe blossfeldiana)、裂叶牵牛 (Pharbitis nil) 和苍耳 (Xanthium pensylvanicum) 等 4 种短日照植物, 以及 4 种长日照 植物欧著草 (Achillea millefolium) 、疗喉草( Trachelium caeruleum) 、意大利风铃草 (Campanula isophylla) 和翠 菊( Callistephus chinensis )) 开花时间的影响，结果发 现, 长日照植物均相应提早 5 14 d 进入开花期, 而 与长日照植物相比, 短日照植物则推迟 $1 \sim 4 \mathrm{~d}$ 开 花。更有趣的是, Mortensen(1985) 认为, 即使同一种 植物, 对 $\mathrm{CO}_{2}$ 浓度的增加也有不同响应。譬如短日 照植物一品红 (Euphorbia pulcherrima) ,当 $\mathrm{CO}_{2}$ 浓度 升高到 $1000 \mu \mathrm{mol} \mathrm{mol}{ }^{-1}$ 时, 可提前 $4.6 \mathrm{~d}$ 开花; 如果 在此基础上再增加 $40 \mu \mathrm{mol} \mathrm{m} \mathrm{m}^{-2} \mathrm{~s}^{-1}$ 的光合光子通 量 ( Photosynthetic photon flux), 则开花还可提前 $6.6 \mathrm{~d}$ 。

植物生殖的起始期，是其生活史中的关键参数， 在成熟时对应年龄或植株大小均反映了生活史的适 合度 (Fitness)（Wesselingh et al，1997)。通常，植物 生殖起始期的提前可通过两种机制来实现:第一是 在植物有性生殖开始时所需最小形体大小保持不变 情况下, 通过提高植物相对生长速率，以加快个体发 育, 使得植株在较短时间内达到有性生殖所需的形 体大小 (Reekie et al , 1994 ;Ladeau \& Clark ,2001; Wagner et al. 2001) 第二是改变植物开花时所需的 形体大小 (Reekie et al.,1994;He \& Bazzaz ,2003)。 而大多数研究者认为, 植物形体的大小是影响植物 开花的首要因素，而植物开花的时间与其年龄关系 不大(Reekie et al. 1994 ;Ladeau \& Clark 2001 ;Wagner et al .2001; ; He \& Bazzaz 2003)。高浓度 $\mathrm{CO}_{2}$ 通过 提高美国商陆 (Phytolacca americana) 相对生长速率 来加快个体发育, 使得该植物提前一周开花, 但该物 种在有性生殖开始时的形体大小并未受到高浓度 $\mathrm{CO}_{2}$ 的影响 ( $\mathrm{He} \&$ Bazzaz ,2003)。1 $000 \mu \mathrm{mol} \mathrm{mol} \mathrm{l}^{-1}$ $\mathrm{CO}_{2}$ 虽然使得拟南芥提前 $5 \mathrm{~d}$ 抽苔, 但此时植株形体 
大小与生长在 $360 \mu \mathrm{mol} \mathrm{mol}{ }^{-1} \mathrm{CO}_{2}$ 浓度条件下植株 抽苔时形体大小无多大差异; 高浓度 $\mathrm{CO}_{2}$ 并不影响 拟南芥开花时植株形体大小, 而主要是通过提高个 体相对生长速率来促进植株提前开花 (Gibeaut et $a l$. ,2001)。另外, Reekie 等 (1991) 通过对 Guara brachycarpa、天人菊 ( Gailardia pulchella)、月见草 (Oenothera laciniata) 和羽扇豆 (Lupinus texensis) 等 4 种植物的研究结果证实, 在 $\mathrm{CO}_{2}$ 浓度增加的条件下， 植物生长和开花起始期都将受到影响, 即植物开花 可能处于不同年龄, 而此时植株形体的大小或多或 少基本一致。因此，一些学者认为, 植物在高浓度 $\mathrm{CO}_{2}$ 生长条件下提前开花, 可能是由于在植株开花 时形体大小保持不变的情况下, 光合速率增加使得 相对生长速率加快, 加速个体发育进程, 促使生殖起 始期所需植株的最小形体时间变短, 从而提前开花, 这属于间接效应 (Ladeau \& Clark,2001; Wagner et $a l$. 2001; He \& Bazzaz ,2003)。但也有一些学者则认 为, 可能是诱导开花激素浓度的改变, 似与生长速率 的加快无直接联系, 尤其是当温度、光周期等参数基 本一致时, $\mathrm{CO}_{2}$ 浓度也可能成为一种直接诱导的因 子(Reekie \& Bazzaz ,1991)。

\section{2 对花、果实与种子产量的影响}

通常，植物花部的发育对 $\mathrm{CO}_{2}$ 浓度变化的响应 相当敏感, 如在高 $\mathrm{CO}_{2}$ 浓度条件下, 大多数植物的开 花数量增多 (Jablonski et al. 2002)。Thurig 等 (2003) 通过对 61 种植物在高浓度 $\mathrm{CO}_{2}$ 下开花影响的研究 时发现, 这些植物在群体水平上, 其花枝数目平均提 高 24\%左右。Jablonski 等 (2002) 对已发表的 159 篇 论文, 涉及 79 个物种的相关资料进行整合分析 (Meta-analysis) 后表明, 在 $500 \sim 800 \mu \mathrm{mol} \mathrm{mol}^{-1} \mathrm{CO}_{2}$ 浓度下, 所有植物的开花数量平均增加 $19 \%$ 。花数 量增加与质量提高对农牧作物和园艺植物来说尤为 重要, 特别是农牧作物开花数量的增加是籽粒增产 的前提条件, 如水稻在 $\mathrm{CO}_{2}$ 浓度升高情况下, 其分藍 和开花数量均有显著增加 (Costa et al. 2003)。同样 对一些园艺植物来说, 花器官数量增加与质量改善, 不仅可增加园艺植物果实产量, 而且还可提高一些 花卉植物观赏效果和切花品质, 从而获取较高的经 济效益。又如以收获果实为目的草莓 (Fragaria ananassa) (Chen et al.,1997)、菜豆 (Phaseolus vulgaris) (Prasad et al. ,2002) 和西红柿 (Lycopersicon esculentum) (Reinert et al. ,1997) 等园艺植物，其开花数 量都有明显增加，同时盛花期间以及开花持续时间
均得到延长, 最终果实产量也相应地得到显著增加。 而对生长在高浓度 $\mathrm{CO}_{2}$ 条件下的一些观赏植物, 如 百合 (Lilium dauricum) (魏胜林等,2001)、蝴蝶兰 (Phalaenopsis) (Endo \& Ikushima,1997) 以及唐菖蒲 (Gladiolus hybridus) (张效平, 1995) 等, 高浓度 $\mathrm{CO}_{2}$ 不 仅能够增加鲜花产量, 而且还明显延长了切花瓶插 的寿命, 进而提高了切花的经济价值。切花瓶插寿 命的提高, 可能与花卉组织中一些抗衰老物质的增 加有关, 如在高浓度 $\mathrm{CO}_{2}$ 条件下百合叶片中脯氨酸、 可溶性糖以及氨基酸含量明显增加, 这些物质是细 胞渗透调节与代谢所必需, 同时还能提供更多能量 物质来延长鲜切花的寿命(魏胜林等 2001)。

关于 $\mathrm{CO}_{2}$ 浓度升高对植物有性生殖产量的影 响，多见于对农作物的研究报道。在通常情况下， $\mathrm{CO}_{2}$ 浓度升高主要是通过增加农作物果实或种子数 量来提高产量, 而对其大小却影响不大 (Ainsworth et al.,2002 ;Jablonski et al .,2002)。Kimball ( 1983 ) 对 430 篇有关农作物的研究报道进行了分析后发现， 农作物平均产量增加约 $34 \%$, 而籽粒平均大小几乎 没有变化。Ainsworth 等(2002)采用整合分析方法， 将 1980 到 2000 年的 20 年间,在国际期刊上发表的 111 篇论文中有关 $\mathrm{CO}_{2}$ 浓度升高对大豆生长影响作 了全面分析, 结果表明, 大豆种子产量平均增加 $24 \%$, 主要是豆荚数目平均增加 $19 \%$ 所致, 而单粒 种子的平均重量并无显著变化。最近, Jablonski 等 (2002) 也发现, 在高浓度 $\mathrm{CO}_{2}$ 处理下, 水稻、大豆、小 麦以及玉米等农作物果实、种子数量和产量平均分 别增加了 $18 \%$ 、16\%和 $25 \%$, 而单粒种子平均重量 仅增加为 $4 \%$ 。除农作物以外, 如白三叶等一些重 要牧草, 在 $\mathrm{CO}_{2}$ 浓度升高条件下, 其种子数量增加 $18 \%$ 以上, 但种子千粒重却未受到 $\mathrm{CO}_{2}$ 的影响 (Wagner et al. 2001)。为何 $\mathrm{CO}_{2}$ 浓度变化对种子数 量的影响程度大于种子大小呢? 通常认为, 植物子 代的适合度与亲代投入的资源量成正相关，但是种 子变大将会使得种子数量下降, 因为植物投入到有 性生殖的资源是有限的, 即种子大小与数量之间存 在着权衡关系 (Trade-off) (Smith \& Fretwell , 1974; Lloyd , 1987)。经典的 Smith-Fretwell 模型成功地解 释了为何种子大小往往保持恒定值, 该模型认为, 植 物子代个体的适合度曲线与其最优种子大小 (Optimal seed size) 密切相关。在一个种群内, 其种子适合 度曲线往往受生境条件影响较小, 这样, 种子大小就 不易发生改变。如果该种群投入到种子生产的资源 增加, 由于种子适合度曲线变化不大, 使得种子大小 
变化也不大,因此, 种子数量将会增加 (Smith \& Fretwell，1974; 张大勇,2004)。 $\mathrm{CO}_{2}$ 浓度升高通常 会提高植物绝对生殖产量, 由于最优种子大小一般 受环境条件(包括 $\mathrm{CO}_{2}$ 浓度变化) 影响较小,所以种 子数量就会相应增加。

因此，在高 $\mathrm{CO}_{2}$ 浓度条件下，通常大多数植物的 生殖生物量, 如花、果实与种子数量等均会显著增 加, 而种子平均大小的增加幅度却相对较少。此外, 一些花卉植物的观赏效果和切花品质也可得到明显 提高。

\section{3 对不同类型植物有性生殖的影响}

虽然高浓度 $\mathrm{CO}_{2}$ 能提高植物生殖生物量的产 量, 但由于各种植物 (如农作物与野生植物, 豆科植 物、 $\mathrm{C}_{3}$ 与 $\mathrm{C}_{4}$ 植物, 定型植物与不定型植物等) 存在 类型上的差异, 这就使得其生殖产量的变化幅度产 生明显的变化。一般认为, 农作物产量增加幅度要 远远高于野生植物(Evans ,1993; Hall \& Ziska ,2000)。 Jablonski 等(2002) 通过对 159 篇文献进行整合分析 发现，高浓度 $\mathrm{CO}_{2}$ 对农作物与野生植物的总生物量 提高幅度无明显差别 均约为 $31 \%$ 。但对果实和种 子的生物量来说，农作物平均增加 $28 \%$ 和 $21 \%$, 而 野生植物只有 $4 \%$ 左右。该作者认为,由于在 $\mathrm{CO}_{2}$ 浓度增加条件下，农作物分配到有性生殖的资源比 例未发生显著变化, 而野生植物在相同条件下分配 到有性生殖的资源却下降了 $14 \%$ 。为何农作物在 $\mathrm{CO}_{2}$ 浓度升高情况下, 其生殖生物量增加幅度要远 远高于野生植物?一些学者认为, 农作物是经过了 数百年人工选择所篮选出来的最优良品种，它们的 果实或种子无论在产量还是在质量上都达到最优状 态。因此在大气 $\mathrm{CO}_{2}$ 浓度不断增加下，它们会充分 利用 $\mathrm{CO}_{2}$ 进行光合作用, 并把较多的资源用于生殖 生长，从而大大地提高了生殖产量。然而对一些野 生植物来说，由于它们是通过长期自然选择的结果， 其物质资源分配在其生长、生殖、生存以及防御等方 面需要有权衡关系; 同时它们在营养和生殖生长特 性上也比农作物具有更大的可塑性。总之,由于野 生植物比农作物的生长环境差, 并且还存在着许多 难以预测的因素, 因此当这类物种在 $\mathrm{CO}_{2}$ 浓度升高 条件下持续繁衍下去时, 是把大部分资源用于营养 生长、生存以及防御等方面，而不太可能分配更多的 资源用于其生殖生长 (Hall \& Ziska ,2000 ; Jablonski et al . 2002 ;Toshihiko et al .,2003)。

通常， $\mathrm{CO}_{2}$ 浓度升高情况下，豆科植物产量增加
幅度高于非豆科的 $\mathrm{C}_{3}$ 植物, 而 $\mathrm{C}_{4}$ 植物其产量增加 幅度最小(Poorter , 1993 ;Wand et al. ,1999)。Jablonski 等(2002)通过研究发现，豆科植物种子平均产量 显著增加 $37 \%$ 非豆科的 $\mathrm{C}_{3}$ 植物增加 $15 \%$ 左右, 而 $\mathrm{C}_{4}$ 植物则无明显变化。以大豆、小麦和玉米为例, 在高浓度 $\mathrm{CO}_{2}$ 条件下, 平均产量分别增加为 $20 \%$ ， $15 \%$ 和 $5 \%$ 。为何不同类型植物的有性生殖对 $\mathrm{CO}_{2}$ 浓度升高的响应程度为: 豆科植物 > 非豆科 $\mathrm{C}_{3}$ 植物 $>\mathrm{C}_{4}$ 植物? 究其原因, 是因为互科植物具有固氮能 力 即使在 $\mathrm{CO}_{2}$ 浓度升高情况下, 也不会由于氮的缺 乏而使其生长受到限制，但对其它非豆科植物来说， 当它们遇到氮素缺乏时, 其生长在一定程度上则会 受到影响, 生殖产量增加的幅度也比豆科植物低。 另外, $\mathrm{C}_{3}$ 植物进行光合作用固定 $\mathrm{CO}_{2}$ 的酶是核酮糖 二磷酸羧化/加氧酶 (Ribulose bisphosphate carboxylase , Rubisco）, $\mathrm{C}_{4}$ 植物为磷酸烯醇式丙酮酸羧化酶 (Phosphoenolpyruvate carboxylase) 和 Rubisco 两种酶 类, $\mathrm{C}_{4}$ 植物在这两种酶的协同作用下利用空气中 $\mathrm{CO}_{2}$ 的能力要远高于 $\mathrm{C}_{3}$ 植物。相对于 $\mathrm{C}_{4}$ 植物来 说, 目前空气中的 $\mathrm{CO}_{2}$ 浓度还远不能使 $\mathrm{C}_{3}$ 植物 Rubisco 酶得以饱和, 当 $\mathrm{CO}_{2}$ 浓度升高时, $\mathrm{C}_{3}$ 植物光合 作用增加幅度和光呼吸抑制程度均高于 $\mathrm{C}_{4}$ 植物, 因 而 $\mathrm{C}_{3}$ 植物总生物量以及生殖生物量增加幅度均会 相应的高于 $\mathrm{C}_{4}$ 植物 (Poorter, 1993 ; Wand et al. , 1999 ;Ward \& Kelly，2004;牛书丽和蒋高明，2003)。 但是也有一些例外, 如 $\mathrm{C}_{3}$ 植物的水稻平均产量增加 $42 \%$ 其产量增幅大于豆科植物的 $37 \%$, 其原因可 能与在农业上广泛采用高产、半矮化、光合能力极强 且具有较大库强 (Sink strength) 的水稻品种, 同时水 稻又长期生长在有水和高养分条件下有关 (Horie $e t$ al. 2000 ; Jablonski et al. 2002)。

依据植物对 $\mathrm{CO}_{2}$ 浓度变化敏感的程度, 通常可 分成定型植物 (Determinate plants) 和不定型植物 ( Indeterminate plants)两大类 (Lawlor \& Keys ,1993 ; Morison \& Lawlor,1999;He \& Bazzaz,2003)。定型植物是 指在植物生活史中, 其发育过程、生长模式乃至植株 大小、器官数量和库源能力等参数都变化不大的植 物, 如欧洲苍耳 (Xanthium strumarium)、黑麦草和白 车轴草 (Trifolium repens) 等 (Wagner et al.,2001； Lewis et al . 2003) ;而在不定型植物的生活史中，上 述各种参数的变化较大, 如野萝卜 ( Raphanus raphanistrum) 与拟南芥等 (Curtis et al. 1994 ;Ward \& Strain ,1997)。过去的研究显示, $\mathrm{CO}_{2}$ 浓度升高时, 定 型植物的果实和种子数目变化不大 (Navas et al. , 
1997 ; Huxman et al. 1999; Wagner et al. 2001) ,而不 定型植物的果实和种子数目却显著增加 (Curtis et al . 1994; Ward \& Strain ,1997 ;Edwards et al.,2001)。 因此，不定型植物的有性生殖对 $\mathrm{CO}_{2}$ 浓度升高的响 应程度远比定型植物高。究其原因, 可能是定型植 物库源能力不易受到改变, 从而使其生殖产量对 $\mathrm{CO}_{2}$ 浓度升高响应程度较小的缘故 (Morison \& Lawlor , 1999)。

\section{4 对花粉与花蜜产量与质量的影响}

菊科植物的豚草 (Ambrosia artemisiifolia) 是一种 能产生大量花粉的植物, 其花粉是引起人类千草 热” (Hay fever)的主要原因之一。据有关报道, 目前 干草热”患者数量逐年增加与空气中花粉数量的增 加有关, 而花粉数量的增加又与大凡 $\mathrm{CO}_{2}$ 浓度的 升高紧密相关 ( Bagarozzi et al . , 1998 ; Ziska \& Caulfield 2000)。例如 Ziska 和 Caulfield (2000) 比较 了在 600 和 $370 \mu \mathrm{mol} \mathrm{mol}{ }^{-1}$ 两种不同 $\mathrm{CO}_{2}$ 浓度下豚 草花粉的产量, 结果发现, 在 $600 \mu \mathrm{mol} \mathrm{mol}{ }^{-1}$ 生长条 件下,豚草花粉产量为 $370 \mu \mathrm{mol} \mathrm{mol}{ }^{-1}$ 的 2.5 倍。此 外,Aloni 等 (2001) 在研究高温与 $\mathrm{CO}_{2}$ 浓度对牛角椒 (Capsicum annuum) 花粉活力影响时发现, 高温能显 著降低其花粉活力, 而高浓度 $\mathrm{CO}_{2}$ 则减弱高温对花 粉活力的影响。未曾受到高温处理的植株, 其花粉 萌发率是受高温处理植株的 3 倍; 在 $800 \mu \mathrm{mol} \mathrm{mol}{ }^{-1}$ $\mathrm{CO}_{2}$ 浓度生长条件下的辣椒, 当给予同样高温处理 后, 其花粉萌发率与对照均无明显差异。通过进一 步的研究发现, 花粉萌发率的改变与花粉中果糖激 酶 (Fructokinase) 和己糖激酶 (Hexokinase)的活性变化 有关,由于这两种酶在花粉萌发过程中的物质代谢 起了关键性的作用, 因此它们的活性降低将直接导 致花粉萌发率的降低。如果植株在 $800 \mu \mathrm{mol} \mathrm{mol}{ }^{-1}$ $\mathrm{CO}_{2}$ 浓度条件下受到同样高温处理后, 其花粉中的 上述两种酶活性并未受到显著影响，结果花粉萌发 率也未发生明显变化。由此推测, 高浓度 $\mathrm{CO}_{2}$ 可能 通过减弱高温对花粉和花药发育过程中相关酶活性 的影响，从而保持了花粉的活力 (Karni \& Aloni， 2002)。

Osborne 等(1997)报道, 在高浓度 $\mathrm{CO}_{2}$ 下生长的 虫豆 (Vicia faba ) 虽然单花蜜产量无显著变化, 但由 于花量增加 $25 \%$, 同时花的寿命又延长 $17 \%$ ，因而 总的花蜜产量也就明显增加了。旱金莲 (Tropaeolum majus) 生长在 $\mathrm{CO}_{2}$ 浓度为 $750 \mu \mathrm{mol} \mathrm{mol}{ }^{-1}$ 的条件下， 其蜜腺分泌蜜汁的速度明显加快, 而蜜汁中蔗糖和
氨基酸等主要成分的浓度却未发生显著变化 (Lake \& Hughes ,1999)。

\section{5 对种子与果实质量的影响}

通常认为,在 $\mathrm{CO}_{2}$ 浓度升高情况下, 种子中的 $\mathrm{C} / \mathrm{N}$ 比升高、而含氮量、蛋白质和自由氨基酸含量降 低、营养成分下降、千粒重增加以及萌发率和萌发势 加强等 (Jablonski et al . 2002;Barbara et al . 2003; Stiling et al. ,2004; He et al. ,2005)。Kimball 等 (2001)研究表明, 在未来的大气 $\mathrm{CO}_{2}$ 浓度增加条件 下，如果土壤中可用氮的含量得不到补充，那么谷物 中的蛋白质含量就会降低 $39 \%$, 而碳水化合物含量 则相对提高，从而导致谷物营养价值的下降。反之， 如果土壤含有足够氮肥, 那么谷物的质量受到的影 响较小, 蛋白含量也只降低 5\% 左右。最近, Barbara 等(2003)对一些草地植物的研究发现, 在 $\mathrm{CO}_{2}$ 浓度 升高情况下, 这些植物种子的平均重量均有所增加， 种子萌发时间缩短, 但种子的含氮量显著降低。还 有一些学者通过对已发表的 278 篇有关文献的分析 表明 种子氮含量平均降低 $14 \%$, 其中非豆科 $\mathrm{C}_{3}$ 植 物种子的氮含量降低 $16 \%$ 左右, 而豆科植物种子的 氮含量无明显变化。究其原因, 可能是豆科植物根 部具有固氮能力的根瘤菌, 它们能从空气中直接固 定氮素, 进而直接补充植物在高 $\mathrm{CO}_{2}$ 浓度下快速生 长对氮肥的需求 (Cotrufo et al. 1998; Allen \& Boote , 2000 ;Jablonski et al.,2002)。另外,火炬松 (Pinus taeda) 在 $\mathrm{CO}_{2}$ 浓度升高情况下, 其种子萌发率提高 两倍, 萌发时间也提前 $5 \mathrm{~d}$, 幼苗的生长速度加快和 生长势增强, 这就使得它们在群体中具有更强的竞 争力 (Hussain et al.,2001)。但也有研究者发现, 种 子重量增加对幼苗大小并无多大影响，其原因可能 是由于种子含氮量的降低抵消了种子重量增加带给 幼苗的优势 (Steinger et al. 2000)。

大气 $\mathrm{CO}_{2}$ 浓度在 $600 \sim 900 \mu \mathrm{mol} \mathrm{mol} \mathrm{m}^{-1}$ 条件下， 草莓果实平均重量和干物质含量均有所增加, 其中 含糖量显著提高, 糖/酸比也相应增加, 从而果实的 品质得到了明显改善 (Chen et al.,1997)。曼陀罗 (Datura stramonium) 的果实可随着 $\mathrm{CO}_{2}$ 浓度的升高， 重量显著增加, 果皮厚度和果皮表面的针刺数量均 有增加; 同时果实内有毒化合物的含量也随之增加。 上述现象说明 植物可能是为增强适应能力所采取 的一种策略, 通常防御性强的植株后代, 其生存能力 要比增加种子数目的后代强 (Garbutt \& Bazzaz, 1984)。 
小麦和水稻等农作物生长在高浓度 $\mathrm{CO}_{2}$ 条件 下，虽然其产量得到提高，但籽粒中的营养成分却有 所下降, 其中包括氮含量降低、 $\mathrm{C} / \mathrm{N}$ 比上升、人体所 需蛋白质和氨基酸的种类与含量, 以及铁和锌等微 量元素含量均明显下降 (Manderscheid et al.,1995; Seneweera \& Conroy , 1997 ;Ziska et al . 2004)。Seneweera 和 Conroy (1997) 在研究高浓度 $\mathrm{CO}_{2}$ 对水稻籽粒 品质影响时发现，不仅籽粒蛋白含量降低，而且人体 所需的 $\mathrm{Fe}$ 和 $\mathrm{Zn}$ 等微量元素含量也显著下降。Manderscheid 等(1995)报道了生长在高浓度 $\mathrm{CO}_{2}$ 条件下 的两个春小麦和春大麦品种 结果发现，上述 4 种作 物的籽粒蛋白含量降低 4\% 30\% , 而籽粒中的 N、 $\mathrm{Ca}$ 和 $\mathrm{Mg}$ 等大量元素含量分别降低 $30 \% 、 13 \%$ 和 $28 \%$ 此外, 微量元素 $\mathrm{S}$ 的含量也有所降低, 进而影 响到一些含 $\mathrm{S}$ 蛋白质的合成。因此, 人们在衡量 $\mathrm{CO}_{2}$ 浓度升高对农作物产量的影响时, 决不能简单 的以产量的增减作为标准, 还应当考虑谷物增产后 品质发生的改变(蒋高明等, 1997;Blumenthal et al. , 1996 ;Rogers et al. ,1998)。

总之, 在大气 $\mathrm{CO}_{2}$ 浓度升高条件下, 植物果实与 种子的产量通常会有所增加, 种子萌发率与萌发势 也会有所提高 (Hussain et al. 2001 ;Ainsworth et al. , 2002 ;Jablonski et al. 2002 ;Barbara et al.,2003)。但 值得注意的是, 除豆科植物的种子以外, 其它植物的 种子营养成分也会有所降低, 主要表现为 $\mathrm{C} / \mathrm{N}$ 比升 高, 蛋白质和自由氨基酸、以及铁和锌等微量元素的 含量均明显下降(Manderscheid et al. 1995 ;Seneweera \& Conroy , 1997 ; Kimball et al. ,2001 ;Ziska et al. , 2004)。

\section{6 对植物群落构建的影响}

特别值得注意的是, 当大气 $\mathrm{CO}_{2}$ 浓度升高后, 由 于植物有性生殖特性发生了变化, 由此将会引发一 系列不寻常的后果。例如当植物开花期提前或延缓 后, 使许多植物的繁殖成效 (Reproductive success) 受 到影响, 从而改变了一些植物群落构建的布局。其 中最典型的例子是,一些虫媒花植物经过长期演化 和自然选择后, 它们均具有特定类型的昆虫在一定 时间来进行采蜜和传粉, 以帮助这些植物完成授粉 和受精作用。如果当大气 $\mathrm{CO}_{2}$ 浓度升高后, 则会引 起此类植物的开花时间过度提前或推迟, 从而在植 物开花的时候, 那些专为其传粉的昆虫可能尚未出 现 或者已迁徙到其它地方去了, 这样就使得这类植 物种群中的个体授粉几率大为减少, 繁殖力下降。
如果经过几代繁殖后, 将会成为退化种群, 最终将可 能导致这类物种的灭绝 (Garbutt \& Bazzaz,1984; Rusterholz \& Erhardt ,1998)。此外, 在同一个植物群 落内生长的不同植物种群之间, 由于大气 $\mathrm{CO}_{2}$ 浓度 升高, 使得其中一些种群的花量增加, 而另一些种群 的花量相对减少, 这样前者就有可能吸引更多昆虫 来采蜜传粉, 长此以往, 花量相对减少的种群授粉几 率明显下降 种群衰退, 这就使得整个群落的竞争等 级 (Competitive hierarchies) 发生相应的改变 (Reekie \& Bazzaz ,1991 ;Reekie et al .,1994)。

植物种子产量与质量的变化, 对植物群落的变 化也有很大的影响。当大气 $\mathrm{CO}_{2}$ 浓度升高后, 使得 有些类型植物的种子产量增加 种子体积变大 种子 的萌发率和萌发势增强等, 这利于其后代在植物群 落中具有更强的竞争力, 最终有可能成为优势种群。 而另一些植物种类, 由于其种子产量和质量变化不 大，子代在植物群落中的竞争力相对减弱，从而可能 成为弱势种群, 并最终走向灭绝的道路 (Baskin \& Baskin ,1998 ;Gutterman ,2000 ;Stiling et al. ,2004)。 Stiling 等 (2004) 在对一个植物群落中 3 种栎属植物 (Quercus myrtifolia、 $Q$. chapmanii 和 $Q$. geminata) 的 研究发现, 在 $\mathrm{CO}_{2}$ 浓度升高下, 前两者的种子产量显 著增加,而后者种子产量却变化不大 虽然它们的种 子大小与萌发率均未发生明显变化, 但是作者仍认 为, $\mathrm{CO}_{2}$ 浓度升高将会改变这个群落中 3 个物种生 殖产量的比例, 其中 Quercus geminata 的种子产量相 对降低, 而同一个群落中的 $Q$. myrtifolia 和 $Q$. chapmanii 就会占据更多的资源和空间, 并进行大量 繁衍; 同时还由于它们种子质量的提高, 种子萌发率 和萌发势的增加 幼苗生长茁壮均能使它们在该群 落中通过种间生存竞争而获胜成为优势种群。相反 地，由于 $Q$. geminata 的生长资源和空间受到限制， 最终被淘汰 (Edwards et al.,2001; Hussain et al.， 2001)。

另外，雌雄异株植物的生长发育，对 $\mathrm{CO}_{2}$ 浓度升 高的响应也存在较大差异。如 Wang 和 Curtis (2001) 在研究雌雄异株颤杨 (Populus tremuloides) 的 生长发育时, 发现在 $\mathrm{CO}_{2}$ 浓度升高条件下, 虽然雄株 光合作用强度增加的幅度高于雌株, 但由于雌株总 叶面积明显高于雄株, 而呼吸作用强度却低于雄株, 雌株最终的生物量显著高于雄株。2003 年, 这些作 者通过对雌雄异株的叉枝蝇子草 (Silene latifolia) 的 研究进一步发现, 在高浓度 $\mathrm{CO}_{2}$ 条件下,雌性个体总 生物量显著增加, 其中生殖器官生物量的增加幅度 
远高于营养器官生物量; 而雄株生殖器官生物量的 增加幅度则明显低于雌株。他们由此推测, 在未来 大气 $\mathrm{CO}_{2}$ 增加情况下, 由于雌雄个体植株生长发育 所受到的影响不同, 使得用于营养生长和生殖生长 的资源分配比例发生变化，从而有可能改变雌雄个 体的比例, 最终将导致植物繁殖、分布格局以及群落 结构等的变化 (Wang \& Curtis ,2001;Wang \& Griffin， 2003)。

因此, 在未来大气 $\mathrm{CO}_{2}$ 浓度升高情况下, 有些植 物的有性生殖对 $\mathrm{CO}_{2}$ 升高表现出正面效应，从而逐 渐成为植物群落中的优势种群, 而那些对 $\mathrm{CO}_{2}$ 升高 表现出负面效应的种类则成为退化种群, 并最终有 可能被淘汰 致使一些植物群落构建发生明显的变 化 ( Garbutt \& Bazzaz, 1984 ; Baskin \& Baskin ,1998; Rusterholz \& Erhardt ,1998; Gutterman 2000;Stiling et al. 2004)。

\section{7 结 语}

植物有性生殖特性的变化是预测植物对全球气 候变化响应的重要指标之一, 其有性生殖特性对大 气 $\mathrm{CO}_{2}$ 浓度升高的响应主要表现为: 植物相对生长 速率加快, 开花提前; 花、果实与种子等生殖生物量 增加。除豆科植物种子外, 其它植物种子的营养成 分均会有不同程度的降低。不过值得注意的是, 在 以往的研究中仍存在一些不足之处。例如, 有些研 究尚未充分考虑到物种本身的特性, 将长日植物与 短日植物混为一谈, 这就有可能使植物的开花时间 对 $\mathrm{CO}_{2}$ 浓度升高的响应得出互为矛盾的结论。大多 数的报道以开花物候学和种子产量等为指标, 而对 这一过程中的生殖生态与生理生化参数的变化重视 不够。再者, 对植物有性生殖特性响应的研究结论 多建立在短期的观察结果, 或以 $1 \sim 2$ 个生长季(生 殖周期) 的变化模式为基础, 忽视了植物长期在高 $\mathrm{CO}_{2}$ 浓度下具有的反馈作用, 以及 $\mathrm{CO}_{2}$ 浓度变化对 植物可能驯化的影响等。我们认为, 在研究大气 $\mathrm{CO}_{2}$ 浓度升高对植物有性生殖的影响时, 不仅要注 重物种特有习性, 同时还应进行长期或多个世代的 连续试验。针对植物有性生殖特性的变化, 还应该 深入开展包括大小孢子发生、雌雄生殖比例、生殖能 力, 以及导致生殖特性变化的生殖生物学和分子生 理学等方面的研究。

\section{参 考 文 献}

Ainsworth EA, Davey PA, Bernacchi CJ, Dermody OC, Heaton
EA, Moore DJ, Morgan PB, Naidu SL, Yoora HS, Zhu XG, Curtis PS, Long SP (2002). A meta-analysis of elevated [ $\left.\mathrm{CO}_{2}\right]$ effects on soybean (Glycine max) physiology, growth and yield. Global Change Biology, 8, 695-709.

Allen LH, Boote KJ (2000) . Crop ecosystem responses to climatic change: soybean. In: Reddy KR, Hodges HF eds. Climate Change and Global Crop Productivity. CABI Publishing, New York, $133-160$.

Aloni B, Peet M, Pharr M, Karni L (2001). The effect of high temperature and high atmospheric $\mathrm{CO}_{2}$ on carbohydrate changes in bell pepper (Capsicum annuum) pollen in relation to its germination. Physiologia Plantarum, 112, 505-212.

Bagarozzi DA, Potempa J, Travis J (1998). Purification and characterization of an arginine-specific peptidase from ragweed ( $\mathrm{Am}$ brosia artemisiifolia) pollen. American Journal of Respiratory Cell \& Molecular Biology, 18, 363 - 369 .

Barbara T, Christian K, Jurg S (2003). Seed production and seed quality in a calcareous grassland in elevated $\mathrm{CO}_{2}$. Global Change Biology, 9, $873-884$.

Baskin CC, Baskin JM (1998). Seeds: Ecology, Biogeography, and Evolution of Dormancy and Germination. Academic Press, San Diego.

Blumenthal C, Rawson HM, McKenzie E, Gras PW, Barlow EWR, Wrigley CW (1996). Changes in wheat grain quality due to doubling the level of atmospheric $\mathrm{CO}_{2}$. Cereal Chemistry, 73, $762-766$.

Chen K, Hu GQ, Lenz F (1997). Effects of $\mathrm{CO}_{2}$ concentration on strawberry. VI. Fruit yield and quality. Journal of Applied Botany, 71, 195 - 200 .

Costa WAJMD, Weerakoon WMW, Herath HMLK, Abeywardena RMI (2003). Response of growth and yield of rice (Oryza sati$v a)$ to elevated atmospheric carbon dioxide in the subhumid zone of Sri Lanka. Journal of Agronomy \& Crop Science, 189, 83 95 .

Cotrufo MF, Ineson P, Scott A (1998). Elevated $\mathrm{CO}_{2}$ reduces the nitrogen concentration of plant tissues. Global Change Biology, $13,43-54$.

Curtis PS, Snow AA, Miller AS (1994). Genotype-specific effects of elevated $\mathrm{CO}_{2}$ on fecundity in wild radish Raphanus raphanistrum. Oecologia, 97, $101-105$.

Edwards GR, Clark H, Newton PCD (2001). The effects of elevated $\mathrm{CO}_{2}$ on seed production and seedling recruitment in a sheepgrazed pasture. Oecologia, 127, 383-394.

Ellis RH, Craufurd PQ, Summerfield RJ, Roberts EH ( 1995 ). Linear relations between carbon dioxide concentration and rate of development toward flowering in sorghum, cowpea and soybean. Annals of Botany, 75, $193-198$.

Endo M, Ikushima I (1997). Effects of $\mathrm{CO}_{2}$ enrichment on yields and preservability of cut flowers in Phalaenopsis. Journal of the Japanese Society of Horticultural Science, 66, 169 - 174 .

Evans LT (1993) . Crop evolution, adaptation and yield. Cambridge 
University Press, Cambridge, UK.

Fang JY (方精云) (2000). Global Ecology-Climate Change and Ecological Response (全球生态学一气候变化与生态响 应). Higher Education Press, Beijing. (in Chinese)

Garbutt K, Bazzaz FA (1984). The effects of elevated $\mathrm{CO}_{2}$ on plants. III. Flower, fruit and seed production and abortion. New Phytologist, 98, $433-446$.

Gibeaut DM, Cramer GR, Seemann JR (2001). Growth, cell walls, and UDP-Glc dehydrogenase activity of Arabidopsis thaliana grown in elevated carbon dioxide. Journal of Plant Physiology, 158, $569-576$.

Gutterman Y (2000). Maternal effects on seeds during development. In: Fenner M ed. Seeds, the Ecology of Regeneration in Plant Communities. CABI Publishing, New York, 59-84.

Hall AE, Ziska LH (2000). Breeding strategies for the 21st century. In: Reddy KR, Hodges HF eds. Climate Change and Global Crop Productivity. CABI Publishing, New York, 407, 436.

He JS, Bazzaz FA (2003) . Density-dependent responses of reproductive allocation to elevated atmospheric $\mathrm{CO}_{2}$ in Phytolacca americana. New Phytologist, 157, 229 - 239.

He JS, Flynn DFB, Wolfe-Bellin K, Fang J, Bazzaz FA (2005) . $\mathrm{CO}_{2}$ and nitrogen, but not population density, alter the size and C/N ratio of Phytolacca americana seeds. Functional Ecology, $19,437-444$.

Horie T, Baker JT, Nakagawa H, Matsui T, Kim HY (2000) . Crop ecosystem responses to climate change: ice. In: Reddy KR, Hodges HF eds. Climate Change and Global Crop Productivity. CABI Publishing, New York, 81 - 106.

Hussain M, Kubiske ME, Connor KF (2001) . Germination of $\mathrm{CO}_{2}-$ enriched Pinus taeda L. seeds and subsequent seedling growth responses to $\mathrm{CO}_{2}$ enrichment. Functional Ecology, 15, 344 350 .

Huxman TE, Hamerlynck EP, Smith SD (1999). Reproductive allocation and seed production in Bromus madritensis ssp. rubens at elevated atmospheric $\mathrm{CO}_{2}$. Functional Ecology, 13, 769 - 777 .

Idso KE, Idso SB (1994). Plant responses to atmospheric $\mathrm{CO}_{2}$ enrichment in the face of environmental constraints, a review of the past 10 years' research. Agricultural Forest Meteorology, 69 , $153-203$

IPCC (2001) . Contribution of working group 1 to the third assessment report of the inter governmental panel on climate change. In: Houghton JT, Ding Y, Griggs DG, Noguer M, Linden PJ, Xiaosu D eds. Climate Change 2001 : the Scientific Basis. Cambridge: Cambridge University Press.

Jablonski LM, Wang XZ, Curtis PS (2002) . Plant reproduction under elevated $\mathrm{CO}_{2}$ conditions: a meta-analysis of reports on 79 crop and wild species. New Phytologist, 156, 9-26.

Jiang GM (蒋高明), Han XG (韩兴国), Lin GH (林光辉) (1997). Response of plant growth to elevated $\left[\mathrm{CO}_{2}\right]$ : a review on the chief methods and basic conclusions based on experiments in the external countries in past decades. Acta Phytoecologica
Sinica (植物生态学报), 21, 489 - 502. (in Chinese with English abstract)

Jiang GM(蒋高明) (1995). The impact of global increasing of $\mathrm{CO}_{2}$ on plants. Chinese Bulletin of Botany (植物学通报), 12, 1 - 7. (in Chinese with English abstract)

Johannessen MM, Mikkelsen TN, Nersting LG, Gullord M, Bothmer RV, Jorgensen RB (2005) . Effects of increased atmospheric $\mathrm{CO}_{2}$ on varieties of oat. Plant Breeding, 124, $253-256$.

Karni L, Aloni B (2002). Fructokinase and hexokinase from pollen grains of bell pepper ( Capsicum annuum) : possible role in pollen germination under conditions of high temperature and $\mathrm{CO}_{2}$ enrichment. Annals of Botany, 90, 607-612.

Kimball BA (1983) . Carbon dioxide and agricultural yield: an assemblage and analysis of 430 prior observations. Agronomy Journal, 75, $779-788$.

Kimball BA, Morris CF, Pinter PJ Jr, Wall GW, Hunsaker DJ, Adamsen FJ, LaMorte RL, Leavitt SW, Thompson TL, Matthias $\mathrm{AD}$, Brooks TJ (2001). Elevated $\mathrm{CO}_{2}$, drought and soil nitrogen effects on wheat grain quality. New Phytologist, 150, 295 303.

Ladeau SL, Clark JS (2001) . Rising $\mathrm{CO}_{2}$ levels and the fecundity of forest trees. Science, 292, $95-98$.

Lake JC, Hughes L (1999). Nectar production and floral characteristics of Tropaeolum majus L. grown in ambient and elevated carbon dioxide. Annals of Botany, 84, 535-541 .

Lawlor DW, Keys AJ (1993). Understanding photosynthetic adaptation to changing climate. In: Fowden L ed. Plant Adaptation to Environmental Stress. Chapman \& Hall, London, 85-106.

Lawlor DW, Mitchell RAC (1991). The effects of increasing $\mathrm{CO}_{2}$ on crop photosynthesis and productivity: a review of field studies. Plant, Cell and Environment, 14, 807 - 818 .

Lewis JD, Wang XZ, Griffin KL, Tissue DT (2003) . Age at flowering differentially affects vegetative and reproductive responses of a determinate annual plant to elevated $\mathrm{CO}_{2}$. Oecologia, 135, $194-201$.

Lin JX (林金星), Hu YX (胡玉喜), Bai KZ (白克智) (1993). The effects of elevated concentration of carbon dioxide on plants. Journal of Plant Resources and Environment (植物资源与环 境) , 2, 55-61. (in Chinese with English abstract)

Lloyd DG (1987). Selection of offspring size at independence and other size-versus-number strategies. American Naturalist, 129 , $800-817$.

Manderscheid R, Bender JJ, Jäger HJ, Weigel HJ (1995). Effects of season long $\mathrm{CO}_{2}$ enrichment on cereals. II. Nutrient concentrations and grain quality. Agriculture, Ecosystems and Environment, 54, 175 - 185 .

Miglietta F, Magliulo V, Bindi M, Cerio L, Vaccari FP, Loduca V, Peressotti A (1998). Free air $\mathrm{CO}_{2}$ enrichment of potato ( Solanum tuberosum L.): development, growth and yield. Global Change Biology, 4, 163 - 172 .

Morison J, Lawlor DW (1999). Interactions between increasing 
$\mathrm{CO}_{2}$ concentration and temperature on plant growth. Plant, Cell and Environment, 22, 659-682.

Mortensen LM (1985). Effect of $\mathrm{CO}_{2}$ enrichment and supplementary light on growth and flowering of poinsettia, Euphorbia pulcherrima Will. Meld Norg Landbruksogsk, 64, 1-8.

Mortensen LM (1987). Review: $\mathrm{CO}_{2}$ enrichment in greenhouse. Crop response. Scientia Horticulturae, 33, $1-25$.

Murray DR (1997). Carbon Dioxide and Plant Responses. Research Studies Press Ltd, Taunton, UK.

Navas ML, Sonie L, Richarte J, Roy J (1997). The influence of elevated $\mathrm{CO}_{2}$ on species phenology, growth and reproduction in a Mediterranean old-field community. Global Change Biology, 3, $523-530$.

Niu SL(牛书丽), Jiang GM(蒋高明) (2003). Effect of elevated $\mathrm{CO}_{2}$ on legume plants with nitrogen fixation. Acta Phytoecologica Sinica (植物生态学报), 27,844-850. (in Chinese with English abstract)

Osborne JL, Awmack CS, Clark SJ, Williams IH, Mills VC (1997). Nectar and flower production in Vicia faba L. (field bean) at ambient and elevated carbon dioxide. Apidologie, 28, $43-55$.

Poorter H (1993). Interspecific variation in the growth response of plants to an elevated ambient $\mathrm{CO}_{2}$ concentration. Vegetatio, 104, $77-97$.

Prasad PVV, Boote KL, Hartwell A, Jean MGT (2002). Effects of elevated temperature and carbon dioxide on seed-set and yield kidney bean (Phaseolus vulgaris L.). Global Change Biology, $8,710-721$

Reekie EG, Bazzaz FA (1991). Phenology and growth in four annual species grown in ambient and elevated $\mathrm{CO}_{2}$. Canadian Journal of Botany, 69, 2475 - 2481 .

Reekie JYC, Hicklenton PR, Reekie EG (1994) . Effects of elevated $\mathrm{CO}_{2}$ on time of flowering in four short-day and four long-day species. Canadian Journal of Botany, 72, 533 - 538 .

Reinert RA, Eason G, Barton J (1997). Growth and fruiting of tomato as influenced by elevated carbon dioxide and ozone. New Phytologist, 137, $411-420$.

Rogers GS, Gras PW, Batey IL, Milham PJ, Payne L, Conroy JP (1998). The influence of atmospheric $\mathrm{CO}_{2}$ concentration on the protein, starch, and mixing properties of wheat flour. Australian Journal of Plant Physiology, 25, 387 - 393.

Rusterholz HP, Erhardt A (1998). Effects of elevated $\mathrm{CO}_{2}$ on flowering phenology and nectar production of nectar plants important for butterflies of calcareous grasslands. Oecologia, 113, 341 349 .

Seneweera SP, Conroy JP (1997). Growth, grain yield and quality of rice (Oryza sativa L.) in response to elevated $\mathrm{CO}_{2}$ and phosphorus. Soil Science Plant Nutrition, 43, 1131 - 1136.

Smith CC, Fretwell SD (1974). The optimal balance between the size and number of offspring. American Naturalist, 136, $154-$ 166.
Steinger T, Gall R, Schmid B (2000). Maternal and direct effects of elevated $\mathrm{CO}_{2}$ on seed provisioning, germination and seedling growth in Bromus erectus. Oecologia, 123, $475-480$.

Stiling P, Moon D, Hymus G , Drake B (2004). Differential effects of elevated $\mathrm{CO}_{2}$ on acorn density, weight, germination, and predation among three oak species in a scrub forest. Global Change Biology, 10, 228 - 232 .

Thurig B, Konner C , Stocklin J (2003) . Seed production and seed quality in a calcareous grassland in elevated $\mathrm{CO}_{2}$. Global Change Biology, 9, 873 - 884 .

Toshihiko K, Kouki H, Tadaki H (2003) . Reproductive allocation of an annual, Xanthium canadense, at an elevated carbon dioxide concentration. Oecologia, 137, $1-9$.

Uprety DC, Dwivedi N, Jain V, Mohan R, Saxenav DC, Jolly M, Paswan G (2003). Responses of rice cultivars to the elevated $\mathrm{CO}_{2}$. Biologia Plantarum, 46, 35-39.

Wagner J, Lscher A, Hillebrand C, Kobald B, Spitaler N, Larcher W (2001). Sexual reproduction of Lolium perenne L. and Trifolium repens $\mathrm{L}$. under free air $\mathrm{CO}_{2}$ enrichment (FACE) at two levels of nitrogen application. Plant, Cell and Environment, 24, $957-965$.

Wand SJE, Midgley GF, Jones MH, Curtis PS (1999) . Responses of wild $\mathrm{C}_{4}$ and $\mathrm{C}_{3}$ grass (Poaceae) species to elevated atmospheric $\mathrm{CO}_{2}$ concentration: a meta-analytic tests of current theories and perceptions. Global Change Biology, 5, 723-741.

Wang XM (汪杏梅), Bai KZ (白克智), Kuang TY (匡廷云) (1997). Responses of plant dark respiration to doubled $\mathrm{CO}_{2}$ concentration. Acta Botanica Sinica (植物学报) 39, 849 - 854 . (in Chinese with English abstract)

Wang XZ, Curtis PS (2001). Gender-specific variation of Populus tremuloides in its physiological and growth responses to elevated $\mathrm{CO}_{2}$. New Phytologist, 150, 675-684.

Wang XZ, Griffin KL (2003). Sex-specific physiological and growth responses to elevated atmospheric $\mathrm{CO}_{2}$ in Silene latifolia Poiret. Global Change Biology, 9, 612-618.

Ward JK, Kelly JK (2004). Scaling up evolutionary responses to elevated $\mathrm{CO}_{2}$ : lessons from Arabidopsis. Ecology Letters, 7, 427 -440 .

Ward JK, Strain BR (1997). Effects of low and elevated $\mathrm{CO}_{2}$ partial pressure on growth and reproduction of Arabidopsis thaliana from different elevations. Plant, Cell and Environment, 20, 254 -260 .

Wei SL(魏胜林), Liu YH(刘业好), Qu HY (屈海泳), Fu SL (傅松玲), Fu YL(傅玉兰) (2001). Effects of high $\mathrm{CO}_{2}$ concentration on physiological and biochemical processes lily (Lilium dauricum). Acta Phytoecologica Sinica (植物生态学报), 25, 410 - 413. (in Chinese with English abstract)

Wesselingh RA, Renate A, Klinkhamer PGL, De JTJ, Boorman LA (1997). Threshold size for flowering in different habitats: effects of size-dependent growth and survival. Ecology, 78, 2118 2132 . 
Yang JY (杨金艳), Yang WQ (杨万勤), Wang KY (王开运) (2002). Effects of interactions between elevated $\left[\mathrm{CO}_{2}\right]$ and increased temperature on growth of plants. Chinese Journal of Applied and Environmental Biology (应用与环境生物学报), 8, 319 - 324. (in Chinese with English abstract)

Zhang DY (张大勇) (2004). The evolution of life history. In: Zhang DY (张大勇) ed. The Evolution of Plant Life History and Reproductive Ecology (植物生活史进化与繁殖生殖生态学). Science Press, Beijing, 1-78. (in Chinese)

Zhang XP(张效平) (1995). Effect of light intensity and $\mathrm{CO}_{2}$ supplement growth and flowering of Gladiolus. Acta Horticulturae Sinica (园艺学报), 22, 73-76. (in Chinese with English abstract)

Zhang XS (张新时), Gao Q (高琼), Yand DA (杨奠安), Zhou
GS (周广胜), Ni J (倪健), Wang Q(王权)（1997）. A gradient analysis and prediction on the Northeast China Transect (NECT) for global change study. Acta Botanica Sinica (植物学 报), 39, $785-799$. (in Chinese with English abstract)

Ziska LH, Caulfield FA (2000). Rising $\mathrm{CO}_{2}$ and pollen production of common ragweed (Ambrosia artemisiifolia), a known allergyinducing species: implications for public health. Australian Journal of Plant Physiology, 27, 893-898.

Ziska LH, Morris CF, Goins EW (2004) . Quantitative and qualitative evaluation of selected wheat varieties released since 1903 to increasing atmospheric carbon dioxide: can yield sensitivity to carbon dioxide be a factor in wheat performance? Global Change Biology, 10, 1810 - 1819 . 